\title{
SEMI-KAEHLERIAN SUBMANIFOLDS OF AN INDEFINITE COMPLEX SPACE FORM
}

\author{
Dedicated to Professor Shingo Murakami on his 60th birthday
}

By Reiko Aiyama, Hisao Nakagawa and Young Jin Suh

\begin{abstract}
The purpose of this paper is to study several classes of semi-Kaehlerian submanifolds of an indefinite complex space form.
\end{abstract}

\section{Introduction.}

An indefinite Kaehlerian manifold of constant holomorphic sectional curvature is called an indefinite complex space form. Montiel and Romero [9] investigated indefinite complex Einstein hypersurfaces of an indefinite complex space forms and showed that totally geodesic indefinite complex hypersurfaces $P_{s}^{n}\left(c^{\prime}\right)$, $C_{s}^{n}, H_{s}^{n}\left(-c^{\prime}\right)$ and an indefinite complex quadric $Q_{s}^{n}$ are those examples. Ikawa, Romero and one of present authors [6] have also shown that by using an indefinite Segre imbedding there exists a product of complex hyperbolic spaces which becomes an example of space-like Einstein-Kaehlerian submanifolds of an indefinite complex hyperbolic space.

Recently, concerning with the study of Calabi's classification [5] for Kaehlerian imbeddings of complex space forms into complex space forms, Romero [18] and Umehara [21] have independently found that there exists a strongly full holomorphic isometric immersion of indefinite complex space forms into indefinite complex space forms.

From this point of view the purpose of this paper is to study several classes of complete semi-Kaehlerian submanifolds of an indefinite complex space form $M_{s+t}^{n+p}\left(c^{\prime}\right)$.

In the first section, the brief summary of indefinite complex submanifolds of an indefinite Kaehlerian manifold are recalled.

The examples of space-like complex Einstein submanifolds of indefinite complex space forms are given in $\S 2$. $\S 3$ is devoted to the study of the spacelike complex submanifolds with constant scalar curvature of $M_{p}^{n+p}\left(c^{\prime}\right)$. In particular, by estimating the scalar curvature and by using Nishikawa's theorem [12], we shall characterize space-like Einstein submanifolds in the case of $c^{\prime}<0$.

Received December 8, 1987 
In $\S 4$, by developing of Omori's theorem [13] one shows that complete complex submanifolds with constant scalar curvature of $M^{n+p}\left(c^{\prime}\right), c^{\prime}>0$, are Einstein if the Ricci tensor and any shape operator is commutative. In $\S 5$ we confine our attention to the indefinite complex space forms $M_{s+t}^{n+p}\left(c^{\prime}\right), c^{\prime} \neq 0$. By virtue of an example in $\S 2$ the local version of Romero's [18] and Umehara's [21] results can be treated. In general, the shape operator of indefinite complex hypersurfaces of $M_{s+t}^{n+1}\left(c^{\prime}\right)$ is not necessarily diagonalizable. Thus $\S 6$ is devoted to the investigation of proper indefinite complex Einstein hypersurfaces of $M_{s+t}^{n+1}\left(c^{\prime}\right)$, which is the local version of Montiel and Romero [9].

It has been proved by Ryan [19] that the complex hypersurface of complex space forms $M^{n+1}\left(c^{\prime}\right), c^{\prime} \neq 0$, which satisfies $R S=0$ is Einstein. On the other hand, Takahashi [20] has shown that it is cylindrical in the case of complex Eucidean space $C^{n+1}$. In $\S 7$ we shall show that there exist many indefinite complex hypersurfaces of an indefinite complex Euclidean space $C_{s+t}^{n+1}$ satisfying $R S=0$ which are not Einstein and not cylindrical.

\section{Indefinite complex submanifolds.}

This section is concerned with indefinite complex submanifolds of an indefinite Kaehlerian manifold. Let $M^{\prime}$ be an $(n+p)$-dimensional connected Kaehlerian manifold of index $2(s+t)(n \geqq 2,0 \leqq s \leqq n, 0 \leqq t \leqq p)$ and let $M$ be an $n$-dimensional connected indefinite complex submanifold of index $2 s$ of $M^{\prime}$. Let $M$ be an indefinite Kaehlerian submanifold of $M^{\prime}$. We choose a local unitary frame field $\left\{E_{A}\right\}=\left\{E_{1}, \cdots, E_{n+p}\right\}$ on a neighborhood of $M^{\prime}$ in such a way that, restricted to $M, E_{1}, \cdots, E_{n}$ are tangent to $M$ and the others are normal to $M$. Here and in the sequel the following convention on the range of indices are used throughout this paper, unless otherwise stated:

$$
\begin{aligned}
& A, B, \cdots=1, \cdots, n, n+1, \cdots, n+p, \\
& i, \jmath, \cdots=1, \cdots, n, \\
& x, y, \cdots=n+1, \cdots, n+p .
\end{aligned}
$$

With respect to the frame field, let $\left\{\omega_{A}\right\}=\left\{\omega_{i}, \omega_{y}\right\}$ be its dual frame field. Then the Kaehlerian metric tensor $g^{\prime}$ of $M^{\prime}$ is given by $g^{\prime}=2 \sum \varepsilon_{A} \omega_{A} \otimes \omega_{A}$. The connection forms on $M^{\prime}$ are denoted by $\omega_{A B}$. The canonical forms $\omega_{A}$ and the connection forms $\omega_{A B}$ of the ambient space satisfy the structure equations

$$
\begin{aligned}
& d \omega_{A}+\sum \varepsilon_{B} \omega_{A B} \wedge \omega_{B}=0, \quad \omega_{A B}+\bar{\omega}_{B A}=0, \\
& d \omega_{A B}+\sum \varepsilon_{C} \omega_{A C} \wedge \omega_{C B}=\Omega^{\prime}{ }_{A B}, \quad \Omega^{\prime}{ }_{A B}=\sum \varepsilon_{C} \varepsilon_{D} R^{\prime}{ }_{A B C \bar{D}} \omega_{C} \wedge \bar{\omega}_{D},
\end{aligned}
$$

where $\Omega^{\prime}{ }_{A B}$ (resp. $R^{\prime}{ }_{\bar{A} B C \bar{D}}$ ) denotes the Riemannian curvature form (resp. the components of the Riemannian curvature tensor $R^{\prime}$ ) on $M^{\prime}$.

Restricting these forms to the submanifold $M$, we have 


$$
\omega_{x}=0
$$

and the induced indefinite Kaehlerian meric $g$ of index $2 s$ of $M$ is given by $g=2 \sum \varepsilon_{j} \omega_{j} \otimes \omega_{j}$. Then $\left\{E_{j}\right\}$ is a local unitary frame field with respect to this metric and $\left\{\omega_{j}\right\}$ is a local dual field due to $\left\{E_{j}\right\}$, which consists of complexvalued 1-forms of type $(1,0)$ on $M$. Moreover $\omega_{1}, \cdots, \omega_{n}, \bar{\omega}_{1}, \cdots, \bar{\omega}_{n}$ are linear independent, and they are cannonical forms on $M$. It follows from (1.3) and the Cartan lemma that the exterior derivatives of (1.3) give rise to

$$
\omega_{x \imath}=\sum \varepsilon_{j} h_{\imath j}^{x} \omega_{j}, \quad h_{\imath \jmath}^{x}=h_{j i}^{x} .
$$

The quadratic form $\Sigma \varepsilon_{i} \varepsilon_{j} \varepsilon_{x} h_{\imath j}^{x} \omega_{i} \otimes \omega_{j} \otimes E_{x}$ with values in the normal bundle is called the second fundamental form of the submanifold $M$. From the structure equations of $M^{\prime}$ it follows that the structure equations for $M$ are similarly given by

$$
\begin{aligned}
& d \omega_{\imath}+\sum \varepsilon_{j} \omega_{i \jmath} \wedge \omega_{j}=0, \quad \omega_{i j}+\bar{\omega}_{j_{\imath}}=0, \\
& d \omega_{i j}+\sum \varepsilon_{k} \omega_{i k} \wedge \omega_{k \jmath}=\Omega_{\imath \jmath}, \quad \Omega_{\imath \jmath}=\sum \varepsilon_{k} \varepsilon_{l} R_{\bar{i}_{j k} i} \omega_{k} \wedge \bar{\omega}_{l},
\end{aligned}
$$

where $\Omega_{\imath \jmath}$ (resp. $R_{i j_{k l}}$ ) denotes the Riemannian curvature form (resp. the components of the Riemannian curvature tensor $R$ ) on $M$. Moreover, the following relationships are defined:

$$
d \omega_{x y}+\sum \varepsilon_{z} \omega_{x z} \wedge \omega_{z y}=\Omega_{x y}, \quad \Omega_{x y}=\sum \varepsilon_{k} \varepsilon_{l} R_{\bar{x} y k i} \omega_{k} \wedge \bar{\omega}_{l},
$$

where $\Omega_{x y}$ is called the normal curvature form of $M$. For the Riemannian curvature tensors $R$ and $R^{\prime}$ of $M$ and $M^{\prime}$ respectively, it follows from (1.4) and (1.6) that we have the Gauss equation

$$
R_{i j k i}=R^{\prime}{ }_{i j k i}-\sum \varepsilon_{x} h_{j k}^{x} \bar{h}_{\imath l}^{x} .
$$

The components of the Ricci tensor $S$ and the scalar curvature $r$ of $M$ are given by

$$
\begin{aligned}
& S_{\imath j}=\sum \varepsilon_{k} R_{j_{\imath k \bar{k}}}^{\prime}-\sum \varepsilon_{r} \varepsilon_{x} h_{\imath r}^{x} \bar{h}_{r j}^{x}, \\
& r=2 \sum \varepsilon_{j} \varepsilon_{k} R^{\prime}{ }_{j k \bar{k}}-2 h_{2},
\end{aligned}
$$

where $\left(h_{\imath \bar{j}}\right)^{2}=\sum \varepsilon_{k} \varepsilon_{x} h_{i k}^{x} \bar{h}_{k j}^{x}$ and $h_{2}=\sum \varepsilon_{k}\left(h_{k \bar{k}}\right)^{2}$.

The indefinite Kaehlerian manifold $M$ is said to be Einstein, if the Ricci tensor $S$ is given by

$$
S_{\imath j}=r \varepsilon_{i} \delta_{i j} / 2 n
$$

The components $R_{i_{j k} i m}$ and $R_{i j k i \bar{m}}$ (resp. $S_{i \bar{j} k}$ and $S_{i j \bar{k}}$ ) of the covariant derivative of the Riemannian curvature tensor $R$ (resp. the Ricci tensor $S$ ) are defined by 


$$
\begin{aligned}
& \sum \varepsilon_{m}\left(R_{\bar{\imath} j_{k i} m} \omega_{m}+R_{\bar{i} j k \bar{l} \bar{m}} \bar{\omega}_{m}\right)=d R_{\bar{\imath} j k i}-\sum \varepsilon_{m}\left(R_{\bar{m} j k i} \bar{\omega}_{m \imath}\right. \\
& \left.+R_{\bar{\imath} m k i} \omega_{m j}+R_{\bar{\imath} \jmath m i} \omega_{m k}+R_{i \jmath k \bar{m}} \bar{\omega}_{m l}\right), \\
& \sum \varepsilon_{k}\left(S_{i j k} \omega_{k}+S_{i j \bar{k}} \bar{\omega}_{k}\right)=d S_{i j}-\sum \varepsilon_{k}\left(S_{k j} \omega_{k i}+S_{i \bar{k}} \bar{\omega}_{k \jmath}\right) \text {. }
\end{aligned}
$$

The second Bianchi formula is given by

$$
R_{i j_{k i m}}=R_{\bar{\imath} \jmath i_{k} k}
$$

and hence we have

$$
S_{i \bar{j} k}=S_{k \bar{j} i}=\sum \varepsilon_{l} R_{\bar{j} i k i l}, \quad r_{\jmath}=2 \sum \varepsilon_{k} S_{j \bar{k} k},
$$

where $d r=\sum \varepsilon_{j}\left(r_{j} \omega_{j}+\bar{r}_{j} \bar{\omega}_{j}\right)$.

Now, the components $h_{\imath j k}^{x}$ and $h_{\imath j \bar{k}}^{x}$ of the covariant derivative of the second fundamental form of $M$ are given by

$$
\sum \varepsilon_{k}\left(h_{i j k}^{x} \omega_{k}+h_{i j k}^{x} \bar{\omega}_{k}\right)=d h_{i j}^{x}-\sum \varepsilon_{k}\left(h_{k j}^{x} \omega_{k i}+h_{i k}^{x} \omega_{k j}\right)+\sum \varepsilon_{y} h_{i j}^{y} \omega_{x y} .
$$

Then, substituting $d h_{\imath \jmath}^{x}$ in this definition into the exterior derivative of (1.4), we have

$$
h_{\imath j k}^{x}=h_{j i k}^{x}=h_{i k \jmath}^{x}, \quad h_{\imath j \bar{k}}^{x}=-R_{\bar{x} \imath j \bar{k}}^{\prime} .
$$

Similarly the components $h_{\imath j k l}^{x}$ and $h_{\imath j k i}^{x}$ of the covariant derivative of $h_{\imath j k}^{x}$ can be defined by

$$
\begin{aligned}
\sum \varepsilon_{l}\left(h_{\imath j k l}^{x} \omega_{l}+h_{i j k i}^{x} \bar{\omega}_{l}\right)= & d h_{i j k}^{x}-\sum \varepsilon_{l}\left(h_{l j k}^{x} \omega_{l i}+h_{i l k}^{x} \omega_{l j}+h_{i j l}^{x} \omega_{l k}\right) \\
& +\sum \varepsilon_{y} h_{i j k}^{y} \omega_{x y},
\end{aligned}
$$

and the simple calculation gives rise to

$$
\begin{aligned}
& h_{\imath j k l}^{x}=h_{\imath j l k}^{x}, \\
& h_{\imath j k \bar{l}}^{x}-h_{\imath j \bar{l}}^{x}=\sum \varepsilon_{r}\left(R_{i_{k i \bar{r}}} h_{r j}^{x}+R_{i_{k j \bar{r}}} h_{\imath r}^{x}\right)-\sum \varepsilon_{y} R_{\bar{x} y k i} h_{i j}^{y} .
\end{aligned}
$$

A plane section $P$ of the tangent space $T_{x} M$ of $M$ at any point $x$ is said to be non-degenerate, provided that $g_{x} \mid T_{x} M$ is non-degenerate. It is easily seen that $P$ is non-degenerate if and only if it has a basis $\{u, v\}$ such that $g(u, u) g(v, v)-g(u, v)^{2} \neq 0$, and a holomorphic plane spanned by $u$ and $J u$ is non-degenerate if and only if it contains some $v$ with $g(v, v) \neq 0$. The sectional curvature of the non-degenerate holomorphic plane $P$ spanned by $u$ and $J u$ is called the holomorphic sectional curvature, which is denoted by $H(P)=H(u)$. The indefinite Kaehlerian manifold $M$ is said to be of constant holomorphic sectional curvature if its holomorphic sectional curvature $H(P)$ is constant for all $P$ and for all points of $M$. Then $M$ is called an indefinite complex space form, which is denoted by $M_{s}^{n}(c)$, provided that it is of constant holomorphic sectional 
curvature $c$, of complex dimension $n$ and of index $2 s$. The standard models of indefinite complex space forms are the following three kinds which are given by Barros and Romero [3] and Wolf [22]: the indefinite complex Euclidean space $C_{s}^{n}$, the indefinite complex projective space $P_{s}^{n} C$ or the indefinite complex hyperbolic space $H_{s}^{n} C$, according as $c=0, c>0$ or $c<0$. For an integer $s$ $(0<s<n)$ it is seen by [3] and [22] that they are only complete, simply connected and connected indefinite complex space forms of dimension $n$ and of index $2 s$.

Now, the Riemannian curvature tensor $R_{i j_{k i}}$ of $M_{s}^{n}(c)$ is given by

$$
R_{\bar{\imath} j k i}=c \varepsilon_{j} \varepsilon_{k}\left(\delta_{i j} \delta_{k l}+\delta_{\imath k} \delta_{j l}\right) / 2 .
$$

In particular, let the ambient space be an indefinite complex space form $M_{s+t}^{n+p}\left(c^{\prime}\right)$ of constant holomorphic sectional curvature $c^{\prime}$. Then we get

$$
\begin{aligned}
R_{\bar{\imath} j k l}= & c^{\prime} \varepsilon_{j} \varepsilon_{k}\left(\delta_{i j} \delta_{k l}+\delta_{i k} \delta_{j l}\right) / 2-\sum \varepsilon_{x} h_{j k}^{x} \bar{h}_{i l}^{x}, \\
S_{\imath j}= & (n+1) c^{\prime} \varepsilon_{\imath} \delta_{i j} / 2-\left(h_{\imath j}\right)^{2}, \\
h_{\imath j k i}^{x}= & c^{\prime}\left(\varepsilon_{k} h_{\imath \jmath}^{x} \delta_{k l}+\varepsilon_{i} h_{j k}^{x} \delta_{i l}+\varepsilon_{j} h_{k i}^{x} \delta_{j l}\right) / 2 \\
& -\sum \varepsilon_{r} \varepsilon_{y}\left(h_{r i}^{x} h_{j k}^{y}+h_{r j}^{x} h_{k i}^{y}+h_{r k}^{x} h_{i j}^{y}\right) \bar{h}_{r l}^{y} .
\end{aligned}
$$

Functions $h_{4}$ and $A_{2}$ are denoted by $h_{4}=\sum \varepsilon_{i} \varepsilon_{j}\left(h_{i j}\right)^{2}\left(h_{j \bar{i}}\right)^{2}$ and $A_{2}=\sum \varepsilon_{x} \varepsilon_{y} A_{y}^{x} A_{x}$, where $A_{y}{ }^{x}=\sum \varepsilon_{i} \varepsilon_{j} h_{\imath j}^{x} \bar{h}_{i j}^{y}$. Then, by means of (1.18), the Laplacian $\Delta h_{2}$ of the function $h_{2}$ is given by

$$
\Delta h_{2}=(n+2) c^{\prime} h_{2} / 2-\left(2 h_{4}+A_{2}\right)+\sum \varepsilon_{x} \varepsilon_{i} \varepsilon_{j} \varepsilon_{k} h_{\imath j k}^{x} \bar{h}_{\imath j k}^{x} .
$$

\section{Examples of space-like complex Einstein hypersurfaces.}

We give here some examples of space-like complex Einstein submanifolds of an indefinite complex space form.

Example 2.1. The indefinite Euclidean space $C^{n}$ is a totally geodesic complex hypersurface of $C_{1}^{n+1}$ in a natural way.

Example 2.2 [9]. For an indefinite complex projective space $P_{s}^{n+1}(c)$, if $\left\{z_{1}, \cdots, z_{s}, z_{s+1}, \cdots, z_{n+2}\right\}$ is the usual homogeneous coordinate system of $P_{s}^{n+1}(c)$, then for each $j$ fixed, the equation $z_{\jmath}=0$ defines a totally geodesic complex hypersurface identifiable with $P_{s}^{n}(c)$ or $P_{s-1}^{n}(c)$, according as $s+1 \leqq j \leqq n+2$ or $1 \leqq j \leqq s$. This means that $P^{n}\left(c^{\prime}\right)$ is a totally geodesic hypersurface of $P_{1}^{n+1}\left(c^{\prime}\right)$. Taking into account that $H_{s}^{n}\left(-c^{\prime}\right)$ is obtained from $P_{n-s}^{n}\left(c^{\prime}\right)$ reversing the sign of its indefinite Kaehlerian metric, the previous discussion shows that $H_{s}^{n}\left(-c^{\prime}\right)$ is a totally geodesic complex hypersurface of both $H_{s}^{n+1}\left(-c^{\prime}\right)$ and $H_{s+1}^{n+1}\left(-c^{\prime}\right)$. Thus $H^{n}\left(-c^{\prime}\right)$ becomes a space-like complex hypersurface of $H_{1}^{n+1}\left(-c^{\prime}\right)$. 
Example 2.3 [9]. Let $Q_{s}^{n}$ be an indefinite complex hypersurface of $P_{s}^{n+1}\left(c^{\prime}\right)$ defined by the equation

$$
-\sum_{\imath=1}^{s} z_{\imath}^{2}+\sum_{\jmath=s+1}^{n+2} z_{\jmath}^{2}=0
$$

in the homogeneous coordinate system of $P_{s}^{n+1}\left(c^{\prime}\right)$. Then $Q_{s}^{n}$ is a complete complex hypersurface of index $2 s$, and moreover, in the similar way to Kobayashi and Nomizu [7], Chapter 11, Example 10.6 it is Einstein and then the Ricci tensor $S$ satisfies $S=n c^{\prime} g / 2$. Then $Q_{s}^{n}$ can be also considered as an indefinite complex Einstein hypersurface of $H_{s+1}^{n+1}\left(-c^{\prime}\right)$. Namely, the complex quadric $Q^{n}$ is a space-like hypersurface of $H_{1}^{n+1}\left(-c^{\prime}\right)$ and the scalar curvature $r$ is given by $r=-n^{2} c^{\prime}$.

Example 2.4 [6]. For the homogeneous coordinate systems $\left\{z_{1}, \cdots, z_{s}\right.$, $\left.z_{s+1}, \cdots, z_{n+1}\right\}$ of $P_{s}^{n}\left(c^{\prime}\right)$ and $\left\{w_{1}, \cdots, w_{t}, \omega_{t+1}, \cdots, w_{m+1}\right\}$ of $P_{t}^{m}\left(c^{\prime}\right)$, a mapping $f$ of $P_{s}^{n}\left(c^{\prime}\right) \times P_{t}^{m}\left(c^{\prime}\right)$ into $P_{R(n, m, s, t)}^{N(n, m)}\left(c^{\prime}\right)$ with $N(n, m)=n+m+n m, R(n, m, s, t)$ $=s(m-t)+t(n-s)+s+t$ is defined by

where

$$
f(z, w)=\left(z_{a} w_{u}, z_{r} w_{x}, z_{b} w_{y}, z_{s} w_{v}\right)
$$

$$
\begin{aligned}
& a, b, \cdots=1, \cdots, s ; \quad r, s, \cdots=s+1, \cdots, n+1, \\
& x, y, \cdots=1, \cdots, t ; \quad u, v, \cdots=t+1, \cdots, m+1 .
\end{aligned}
$$

Then $f$ is a well defined holomorphic mapping and it is seen by Barros and Romero [3] that $f$ is also an isometric imbedding, which is called an indefinite Segre imbedding. In particular, if $s=t=0$, then $f$ is a classical Segre imbedding (the second author and Takagi [11]). By using that the indefinite complex hyperbolic space $H_{s}^{n}\left(-c^{\prime}\right)$ is obtained by the changing the indefinite Kaehlerian metric of $P_{n-s}^{n}\left(c^{\prime}\right)$ by its negative, another indefinite Segre imbedding

$$
f: H_{s}^{n}\left(-c^{\prime}\right) \times H_{t}^{m}\left(-c^{\prime}\right) \rightarrow H_{S}^{N(n, m, m, s)}\left(-c^{\prime}\right)
$$

is given, where $S(n, m, s, t)=(n-s)(m-t)+s t+s+t$. In particular, for $s=t=0$ we have a holomorphic isometric imbedding $f$ of a product of complex hyperbolic spaces $H^{n}\left(-c^{\prime}\right) \times H^{m}\left(-c^{\prime}\right)$ into an indefinite complex hyperbolic space $H_{n m}^{N(n, m)}\left(-c^{\prime}\right)$.

Example 2.5 [18]. Let $f: M \rightarrow M_{S}^{N}\left(c^{\prime}\right)$ be a holomorphic isometric immersion of an indefinite Kaehlerian manifold $M$ into a complete and simply connected indefinite complex space form $M_{S}^{N}\left(c^{\prime}\right)$. Then $f$ is said to be strongly full if $f(M)$ is not contained in an autoparallel complex submanifold of $M_{S}^{N}\left(c^{\prime}\right)$. It is seen in [18] that $P_{s}^{n}(c)$ admits a strongly full holomorphic isometric immersion into $P_{S}^{N}\left(c^{\prime}\right)$ if and only if $c^{\prime}=k c$ for some positive integer $k, N=\left(\begin{array}{c}n+k \\ k\end{array}\right)-1$ and $S=\sum_{\jmath=0}^{[(k+1) / 2]-1}\left(\begin{array}{c}s+2 j \\ 2 j+1\end{array}\right)\left(\begin{array}{c}n-s+k-2 j-1 \\ k-2 j-1\end{array}\right)$ if $s>0,[(k+1) / 2]$ denoting the 
greatest integer less than or equal to $(k+1) / 2$, and $S=0$ if $s=0$.

Changing the indefinite Kaehlerian metric of $P_{n}^{n}(c)$ by its opposite, we have that there exists a strongly full holomorphic isometric immersion of $H^{n}(-c)$ into $H_{S^{\prime}(n, k)}^{N(n, k)}(-k c)$, where $S^{\prime}(n, k)=N(n, k)-S(n, n, k)$ and $S(n, n, k)$ $=\sum_{j=0}^{[(k+1) / 2]-1}\left(\begin{array}{l}n+2 j \\ 2 j+1\end{array}\right) . \quad$ It is seen that $N(n, 2)-n=S^{\prime}(n, 2)=n(n+1) / 2$ and $N(n, k)-n>S^{\prime}(n, k)$ if $k>2$.

\section{Space-like submanifolds.}

This section is concerned with space-like submanifolds with constant scalar curvature of an indefinite complex space form. Let $M$ be a space-like submanifold of an indefinite complex space form $M^{\prime}=M_{p}^{n+p}\left(c^{\prime}\right)$. First of all, the Laplacian of the square length $h_{2}$ of the second fundamental form is estimated. Since $M$ is space-like, the matrix $\left(\left(h_{j \bar{k}}\right)^{2}\right)$ is a negative semi-definite Hermitian one, whose eigenvalues $\lambda_{j}^{\prime}$ are non-positive real valued functions on $M$. On the other hand, the matrix $\left(A_{y}{ }^{x}\right)$ is by definition a positive semi-definite Hermitian one, whose eigenvalues are denoted by $\lambda_{x}$. Then $\lambda_{x}$ are non-negative and we have

$$
\begin{aligned}
& \left(-h_{2}\right)^{2} \geqq h_{4}=\sum_{x} \lambda_{x}{ }^{2} \geqq\left(-h_{2}\right)^{2} / n, \\
& h_{2}{ }^{2} \geqq A_{2}=\sum_{x} \lambda_{x}{ }^{2} \geqq\left(\sum_{x} \lambda_{x}\right)^{2} / p=h_{2}{ }^{2} / p .
\end{aligned}
$$

Since the Laplacian $\Delta h_{2}$ of $h_{2}$ is given by (1.19), we have

$$
\Delta h_{2} \leqq\left\{n p(n+2) c^{\prime} h_{2}-2(n+2 p) h_{2}{ }^{2}\right\} / 2 n p,
$$

where the equality holds true if and only if

$$
\lambda_{3}=\lambda, \quad \lambda_{x}=\mu \text { for any indices } j \text { and } x
$$

and $h_{j k}^{x}$ are parallel. This means that for a non-negative function $f$ defined by $-h_{2}$ the following inequality

$$
\Delta f \geqq\left\{n p(n+2) c^{\prime} f+2(n+2 p) f^{2}\right\} / 2 n p .
$$

On the other hand, the square of the norm of a tensor

$$
\sum\left\{\varepsilon_{x} h_{j k}^{x} h_{i l}^{x}-h_{2}\left(\delta_{i j} \delta_{k l}+\delta_{i k} \delta_{j l}\right) / n(n+1)\right\}
$$

gives rise to an inequality

$$
A_{2} \geqq 2 h_{2}{ }^{2} / n(n+1),
$$

where the equality holds true if and only if $M$ is of constant holomorphic curvature. From this result it follows that

$$
\Delta f \geqq(n+2)\left\{n(n+1) c^{\prime} f+4 f^{2}\right\} / 2 n(n+1) .
$$


As a direct consequence of these estimates, one finds the following

Proposition 3.1. Let $M$ be a space-like complex submanifold with constant scalar curvature of $M^{\prime}=M_{p}^{n+p}\left(c^{\prime}\right)$.

(1) If $c^{\prime} \geqq 0$, then $M$ is totally geodesic.

(2) If $c^{\prime}<0$ and $r \geqq n^{2}(n+p+1) c^{\prime} /(n+2 p)$, then $M$ is Einstein, $r=n^{2}(n+p+1) c^{\prime}$ $/(n+2 p)$ and the second fundamental form is parallel.

(3) If $c^{\prime}<0$ and $r \geqq n(n+1) c^{\prime} / 2$, then $M$ is a complex space form $M^{n}\left(c^{\prime} / 2\right)$ and $p \geqq n(n+1) / 2$.

Proof. Since the scalar curvature $r$ is constant, it turns out that $f$ is also constant and hence by (3.2) we get

$$
n p(n+2) c^{\prime} f+2(n+2 p) f^{2} \leqq 0,
$$

which means that the first and second assertions are trivial.

The last one is easily derived from (3.3). In this case, the matrix $\left(A_{y}{ }^{x}\right)$ has at most two distinct eigenvalues 0 and $-c^{\prime} / 2$. Since the trace $A_{1}$ of the above matrix is given by $A_{1}=\sum \varepsilon_{x} A_{x}^{x}=h_{2}$, we have $h_{2}=c^{\prime} s / 2$, where $s$ denotes the multiplicity of $-c^{\prime} / 2$, from which it follows that $s=n(n+1) / 2$. q. e.d.

Remark. Examples of space-like Einstein-Kaehlerian submanifolds of an indefinite complex space form $M^{\prime}=M_{p}^{n+p}\left(c^{\prime}\right), c^{\prime}<0$, are given in $\S 2$ :

(1) $M=Q^{n}$ of $H_{p}^{n+p}\left(c^{\prime}\right), p=1, r=n^{2} c^{\prime}$.

(2) $M=H^{n}\left(c^{\prime} / 2\right)$ of $H_{p}^{n+p}\left(c^{\prime}\right), p=n(n+1) / 2, r=n(n+1) c^{\prime} / 2$.

(3) $M=H^{n / 2}\left(c^{\prime}\right) \times H^{n / 2}\left(c^{\prime}\right)$ of $H_{p}^{n+p}\left(c^{\prime}\right), p=n^{2} / 4, r=\{n(n+1)-2 p\} c^{\prime}$.

It was proved in [2] that a complete space-like complex submanifold of $M^{\prime}=M_{p}^{n+p}\left(c^{\prime}\right), \quad c^{\prime} \geqq 0$, is totally geodesic. However, in the case where $c^{\prime}<0$, Example 2.5 shows that there are many complete not Einstein space-like submanifolds. One proves here the following

THEOREM 3.2. Let $M$ be a complete space-like complex submanifold of $M^{\prime \prime}=$ $M_{p}^{n+p}\left(c^{\prime}\right), c^{\prime}<0$.

(1) If $r \geqq n^{2}(n+p+1) c^{\prime} /(n+2 p)$, then $M$ is Einstein, $r=n^{2}(n+p+1) c^{\prime} /(n+2 p)$ and the second fundamental form is parallel.

(2) If $r \geqq n(n+1) c^{\prime} / 2$, then $M$ is an indefinite complex space form $M^{n}\left(c^{\prime} / 2\right)$ and $p \geqq n(n+1) / 2$.

In order to Theorem 3.2, the following theorem due to Nishikawa [12] is needed.

THEOREM (Nishikawa). Let $N$ be a complete Riemannan manifold whose Ricci curvature is bounded from below and let $f$ be a non-negative function. If it satisfies 


$$
\Delta f \geqq k f^{2},
$$

where $k$ is a positive constant, then $f$ vanishes identically on $N$.

Proof of Theorem 3.2. The first assertion is only proved. The other is verified by the same method. Since the right hand side of (3.2) is non-negative, the conclusion is trivial by the maximal principle if $M$ is compact.

Now, $M$ is assumed to be non-compact and complete. For the function $f$ defined by $-h_{2}$ and the negative number $b$ defined by $n p(n+2) c^{\prime} / 2(n+2 p)$ the assumption of the scalar curvature implies that $f \geqq-b>0$. Hence a function $F$ on $M$ defined by $f+b$ satisfies

$$
\Delta F \geqq k F(F-b) \geqq k F^{2},
$$

where $k=(n+2 p) / n p$. Accordingly, the theorem due to Nishikawa yields that $F$ vanishes identically on $M$.

This completes the proof.

COROLLARY 3.3. Let $M$ be a complete space-like complex submanifold of $M^{\prime}=M_{p}^{n+p}\left(c^{\prime}\right), c^{\prime}<0$.

(1) If every Ricci curvature of $M$ is greater than or equal to $n(n+p+1) c^{\prime}$ $/ 2(n+2 p)$, then $M$ is Einstein.

(2) If every Ricci curvature of $M$ is greater than or equal to $(n+1) c^{\prime} / 4$, then $M$ is a complex space form $M^{n}\left(c^{\prime} / 2\right)$.

\section{Complex submanifolds.}

This section is devoted to the investigation of complete complex submanifolds with constant scalar curvature of $M^{\prime}=M^{n+p}\left(c^{\prime}\right)$. Let $M$ be an $n$-dimensional complex submanifold of $M^{\prime}$. The components $S_{\imath j k i}$ and $S_{i j k i}$ of the covariant derivative of $S_{\imath j k}$ are expressed by

$$
\sum\left(S_{i j k l} \omega_{l}+S_{i j k i} \bar{\omega}_{l}\right)=d S_{i j k}-\sum\left(S_{l j k} \omega_{l i}+S_{i \bar{l} k} \bar{\omega}_{l j}+S_{\imath j_{l}} \omega_{l k}\right) .
$$

By the exterior differentiation of the definition of $S_{\imath j_{k}}$ and by taking account of (4.1) the Ricci formula for the Ricci tensor $S$ is given as follows:

$$
S_{i j k i}-S_{\imath j \bar{l} k}=\sum\left(R_{i k i \bar{r}} S_{r \bar{j}}-R_{i k r j} S_{i \bar{r}}\right) .
$$

Assume that the scalar curvature $r$ of $M$ is constant. Since we have $\sum S_{j j_{k}}=0$ by (1.13), it follows from (4.2) that we have

$$
\begin{aligned}
\Delta S_{k \bar{j}} & =\sum\left(S_{k \bar{r}} S_{r \bar{j}}-R_{i_{k} r j} S_{i \bar{r}}\right) \\
& =c^{\prime}\left(2 n S_{k \bar{j}}-r \delta_{k j}\right) / 4-\sum\left\{\left(h_{k \bar{r}}\right)^{2} S_{r \bar{j}}-h_{k r}^{x} \bar{h}_{j s}^{x} S_{s \bar{r}}\right\} .
\end{aligned}
$$

On the other hand, by combining the relation $\sum S_{j \bar{k}} S_{k j}-r^{2} / 4 n=h_{4}-h_{2}{ }^{2} / n$ together with the above equation, the following one 


$$
\begin{aligned}
\Delta\left(h_{4}-h_{2}{ }^{2} / n\right)= & n c^{\prime}\left(h_{4}-h_{2}{ }^{2} / n\right)+2 \sum h_{r s i}^{x} \bar{h}_{r s j}^{y} h_{j t}^{y} \bar{h}_{t \imath}^{x} \\
& -\sum\left(h_{\imath r}^{x} S_{\bar{r} j}-S_{i \bar{r}} h_{r j}^{x}\right)\left(h_{i s}^{x} S_{\bar{s} j}-S_{i \bar{s}} h_{s j}^{x}\right)^{-}
\end{aligned}
$$

is derived. Then we can prove the following

THEOREM 4.1. Let $M$ be a complete complex submanifold with constant scalar curvature of $M^{\prime}=M^{n+p}\left(c^{\prime}\right), c^{\prime}>0$. If the Ricci tensor and any shape operator is commutative, then $M$ is Einstein. $[8]$.

Remark. In the case where $M$ is compact, Theorem 4.1 is proved by Kon

In order to Theorem 4.1, the following theorem due to Omori [13] for the estimate of the Laplacian of the function of class $C^{2}$ is needed. This is slightly different from the original one.

THEOREM (Omori). Let $N$ be a complete Riemannian manifold whose Ricci curvature is bounded from below and let $F$ be a function of class $C^{2}$ on $N$. If $F$ is bounded from below, then for any point $p$ and any $\varepsilon>0$ there exists a point $q$ such that

$$
|\operatorname{grad} F(q)|<\varepsilon, \quad \Delta F(q)>-\varepsilon, \quad F(q) \leqq F(p) .
$$

Proof of Theorem 4.1. A function $f$ is defined by

$$
f=h_{4}-h_{2}{ }^{2} / n \text {. }
$$

Then it follows from (3.1) that $f$ is non-negative and the equality holds true if and only if all eigenvalues of the Hermitian matrix $\left(h_{j_{k}}\right)^{2}$ are equal. By (4.3) and the assumption of the theorem we have

$$
\Delta f \geqq n c^{\prime} f \text {, }
$$

where the equality holds true if and only if $S_{i j k}=0$ for any indices. For any positive constant $a$, a function $F$ defined by $1 /(f+a)^{1 / 2}$ is smooth and bounded. On the other hand, the Ricci curvature of $M$ is given by

$$
S_{j \bar{k}}=\left\{(n+1) c^{\prime} / 2-\lambda_{j}\right\} \delta_{j k},
$$

and since the scalar curvature is constant, $h_{2}=\Sigma \lambda_{3}$ is also constant. By (3.1) we have $h_{4} \leqq h_{2}{ }^{2}$ and hence $h_{4}$ is bounded from above. Hence all eigenvalues $\lambda$, are bounded from above by a positive number $R$, which implies that the Ricci curvature is greater than or equal to

$$
\left\{(n+1) c^{\prime} / 2-R\right\} \text {. }
$$

This means that the theorem due to Omori can be applied to the function $F$. 
For any point $p$ and for any $\varepsilon>0$ there exists a point $q$ at which $F$ satisfies (4.4). It follows from these properties that we have

$$
\varepsilon\{3 \varepsilon+2 F(q)\}>F(q)^{4} \Delta f(q) \geqq 0
$$

by the direct calculation. When $\varepsilon$ tends to 0 , the left hand side converges to 0 , because the function $F$ is bounded. For a convergent sequence $\left\{\varepsilon_{m}\right\}$ such that

$$
\boldsymbol{\varepsilon}_{m} \rightarrow 0 \quad(m \rightarrow 0)
$$

there exists a point sequence $\left\{q_{m}\right\}$ so that the sequence $\left\{\boldsymbol{\varepsilon}_{m}\left(3 \boldsymbol{\varepsilon}_{m}+2 F\left(q_{m}\right)\right\}\right.$ converges to 0 by taking a subsequence if necessary, and hence we have

$$
F\left(q_{m}\right)^{4} \Delta f\left(q_{m}\right) \rightarrow 0 .
$$

On the other hand, by the definition of $F$ it yields

$$
\begin{aligned}
F & =1 /(f+a)^{1 / 2}=1 /\left(h_{4}-h_{2}{ }^{2} / n+a\right)^{1 / 2} \\
& \geqq 1 /\left\{(n-1) h_{2}{ }^{2} / n+a\right\}^{1 / 2} .
\end{aligned}
$$

Accordingly the function $F$ is bounded from below by the positive constant, from which together with the above relation we have

$$
\Delta f\left(q_{m}\right) \rightarrow 0
$$

Then (4.5) implies that the sequence $\left\{f\left(q_{m}\right)\right\}$ converges to 0 and hence the sequence $\left\{F\left(q_{m}\right)\right\}$ converges to $a^{-1 / 2}$. On the other hand, since we have

$$
F\left(q_{m}\right) \leqq F(p) \leqq a^{-1 / 2}
$$

for the fixed point $p$ by (4.4), the point is the maximal one of $F$ and hence

$$
f(p)=0 \text { for any point } p \text {. }
$$

This completes the proof.

Remark. The theorem due to Nishikawa stated in $\S 3$ can be verified using the estimate of the Laplacian of the distance function from a point by Yau [23]. However the above proof suggests another one of Nishikawa's theorem. Namely, it is sufficient if the property (4.7) is derived under the assumption (4.6). For any positive number $\varepsilon$ there is an integer $N_{0}$ such that for any $m \geqq N_{0}$ we have

$$
\boldsymbol{\varepsilon}(f+a)^{2}\left(q_{m}\right)>\Delta f\left(q_{m}\right) \geqq k f^{2}\left(q_{m}\right),
$$

which implies that the sequence $\left\{f\left(q_{m}\right)\right\}$ is bounded and hence $F$ is bounded from below by a positive constant. It turns out that (4.7) holds true.

Complex hypersurfaces of $M^{\prime}=M^{n+1}\left(c^{\prime}\right)$ are next considered. Assume that the scalar curvature $r$ is constant. Then (4.3) is simplified as 


$$
\Delta h_{4}=n c^{\prime}\left(h_{4}-h_{2}{ }^{2} / n\right)+2 \Sigma\left(h_{\imath j}\right)^{2} h_{\jmath r s} \bar{h}_{2 r s} .
$$

On the other hand, since we have

$$
\sum h_{r s i} \bar{h}_{r s j}=-c^{\prime}\left\{h_{2} \delta_{i j}+2\left(h_{i j}\right)^{2}\right\} / 2+\left\{2\left(h_{i j}\right)^{4}+h_{2}\left(h_{i j}\right)^{2}\right\},
$$

because of the constant scalar curvature and (1.18), the above equation is reduced to

$$
\Delta h_{4}=c^{\prime}\left\{(n-2) h_{4}-2 h_{2}{ }^{2}\right\}+2\left(2 h_{6}+h_{2} h_{4}\right),
$$

where $h_{6}=\sum\left(h_{j \bar{r}}\right)^{2}\left(h_{r \bar{s}}\right)^{2}\left(h_{s \bar{j}}\right)^{2}$. For eigenvalues $\lambda_{j}$ of the Hermitian matrix $\left(h_{j \bar{k}}\right)^{2}$, the function $h_{6}$ is given by $\sum \lambda_{j}{ }^{3}$ and hence we have $h_{6} \geqq h_{2} h_{4} / n$. Thus we have

$$
\Delta h_{4} \geqq c^{\prime}(n-4) h_{4}+2(n+2) h_{2} h_{4} / n,
$$

provided that $c^{\prime} \leqq 0$. By means of this inequality, the following theorem for complex hypersurfaces is proved.

THEOREM 4.2. Let $M$ be a complete complex hypersurface of $M^{\prime}=M^{n+1}\left(c^{\prime}\right)$. If the scalar curvature of $M$ is constant, then the following statements hold true:

(1) If $c^{\prime} \geqq 0$, then $M$ is totally geodesic or $S=n c^{\prime} g / 2$, the latter case arising only when $c^{\prime}>0$.

(2) If $c^{\prime}<0$ and if $n \leqq 4$, then $M$ is totally geodesic.

Proof. The case where $c^{\prime}>0$ is a direct consequence of Theorem 4.1. The others are concluded by (4.8) and the same procedure of the proof of Theorem 4.1.

\section{Indefinite complex space forms.}

Extending Calabi's classification [5] for Kaehlerian imbeddings of complete simply connected complex space forms into complete and simply connected complex space forms, Romero [18] and Umehara [21] proved recently the indefinite version independently. In this section the local version of Romero and Umehara's result is treated. The following two results are proved.

THEOREM 5.1. Let $M=M_{s}^{n}(c)$ be an $n$-dimensional indefinite complex space form immersed in $M^{\prime}=M_{s+t}^{n+p}\left(c^{\prime}\right)$.

(1) If $c^{\prime} \neq 0$, then $c^{\prime}=k c$ and $n+p \geqq\left(\begin{array}{c}n+k \\ k\end{array}\right)-1$ for some positive integer $k$.

(2) If $c^{\prime}=0$ if and only if $c=0$.

Proposition 5.2. Let $M=M_{s}^{n}(c)$ be an n-dimensional indefinite complex space form immersed in $M^{\prime}=M_{s+t}^{n+p}\left(c^{\prime}\right), c^{\prime} \neq 0$ and $t=p$.

(1) If $c^{\prime}>0$, then $c^{\prime}=c$ (i.e. $M$ is totally geodesic in $\left.M^{\prime}\right)$.

(2) If $c^{\prime}<0$, then $c^{\prime}=c$ or $2 c$, the first case arising only when $M$ is totally geodesic and the other arising only when $s=0$. 
By the similar direct calculation to that in the local version of Calabi's result by the second author and Ogiue [10], we can obtain the following

LEMMA 5.3.

$$
\sum \varepsilon_{x} h_{\imath_{1} \cdots i_{k}}^{x} \bar{h}_{\jmath_{1} \cdots \jmath_{l}}^{x}=\left\{\begin{array}{lc}
0 & \text { for all } k \neq l, \\
\prod_{r=1}^{k-1}\left(c^{\prime}-r c\right) \varepsilon_{\imath_{1}} \cdots \varepsilon_{\imath_{k}} \sum_{\tau} \delta_{\tau\left(i_{1}\right)_{1}} \cdots \delta_{\tau\left(i_{k}\right) \jmath_{k}} / 2^{k-1} & \text { for } k=l,
\end{array}\right.
$$

where $\Sigma_{\tau}$ denotes the summation on all permutations $\tau$ with respect to indices $i_{1}, \cdots, i_{k}$.

From the second equation of the above lemma we have

$$
\begin{aligned}
& \sum \varepsilon_{x} h_{\imath_{1} \cdots \imath_{k+1}}^{x} \bar{h}_{i_{1} \cdots \imath_{k+1}}^{x} \\
& =\prod_{r=1}^{k}\left(c^{\prime}-r c\right)\left(\varepsilon_{\jmath_{1}}\right)^{n_{1}} \cdots\left(\varepsilon_{\jmath_{a}}\right)^{n} a \sum_{s=1}^{a} n_{s} ! / 2^{k}, n_{1}+\cdots+n_{a}=k+1,
\end{aligned}
$$

where $j_{1}, \cdots, j_{a}$ are distinct indices among $i_{1}, \cdots, i_{k+1}$. Using (5.1) we prove

LEMMA 5.4. (1) If $c^{\prime} \neq 0$, then there exists a positive integer $k$ such that $c^{\prime}=k c$.

(2) $c^{\prime}=0$ if and only if $c=0$.

Proof. For any positive integer $m$, we put $p_{m}=\left(\begin{array}{c}n+m \\ m\end{array}\right)-(n+1)$. Then there exists an integer $m$ such that $p<p_{m}$. Let $H=\left(H_{\mu}^{\lambda}\right)$ be a matrix of order $p_{m}$ defined by

$$
\begin{aligned}
& H_{\mu}^{x-n}= \begin{cases}h_{i_{1} \imath_{2}}^{x} & \text { for } \mu \leqq p_{2}, i_{1} \leqq i_{2}, \\
h_{i_{1} 2_{2} \imath_{3}}^{x} & \text { for } p_{2}<\mu \leqq p_{3}, i_{1} \leqq i_{2} \leqq i_{3}, \\
h_{\imath_{1} \cdots i_{m}}^{x} & \text { for } p_{m-1}<\mu \leqq p_{m}, i_{1} \leqq i_{2} \leqq \cdots \leqq i_{m},\end{cases} \\
& H_{\mu}{ }^{\lambda}=0 \quad \text { for } p<\lambda \leqq p_{m},
\end{aligned}
$$

and let $H^{\prime}=\left(\varepsilon_{\lambda} H_{\mu}{ }^{\lambda}\right)$ be a matrix of order $p_{m}$. By choosing the integer $m$, these two matrices are both singular. On the other hand, we have from (5.1)

$$
{ }^{t} H \cdot \bar{H}^{\prime}=\left(\begin{array}{cc}
\sum \varepsilon_{x} h_{11}^{x} \bar{h}_{11}^{x} & 0 \\
0 & \sum \varepsilon_{x}{\underset{m}{h_{m n}^{x}}}_{\bar{h}_{n \ldots n}^{x}} \underset{m}{x}
\end{array}\right)
$$

and hence at least one entry of the diagonal of the above matrix must be equal to zero, from which together with (5.1) it follows that there exists an integer $k$ so that if $c^{\prime} \neq 0$, then $c^{\prime}=k c$.

The second assertion is trivial by the above argument.

q.e.d. 
Remark. In Theorem 5.1 (2), we do not know whether the submanifold $M=M_{s}^{n}(c)$ is totally geodesic or not.

LEMMA 5.5. If there is a positive integer $k$ such that $c^{\prime}=k c$, then $p \geqq p_{k}$ $=\left(\begin{array}{c}n+k \\ k\end{array}\right)-(n+1)$.

The proof is derived in the proof of Lemma 5.4 .

Let $M=M_{s}^{n}(c)$ be a complex submanifold of $M^{\prime}=M_{s+t}^{n+p}\left(c^{\prime}\right), c^{\prime} \neq 0$. In particular, suppose that $t=p$. Then it follows from (5.1) that we have

$$
-\sum \underset{\underbrace{h_{1}}_{k+1}{\underset{k+1}{1 \cdots 1}}_{k+1}^{x}}{h_{k+1}^{x}}=\prod_{r=1}^{k}\left(c^{\prime}-r c\right)\left(\varepsilon_{1}\right)^{k+1} k ! / 2^{k},
$$

because of $\varepsilon_{x}=-1$ for any index $x$. By the values of $k$ in (5.2) the following relations are given:

$$
\begin{aligned}
& \left(c^{\prime}-c\right) \leqq 0, \\
& \varepsilon_{1}\left(c^{\prime}-c\right)\left(c^{\prime}-2 c\right) \leqq 0, \\
& \quad\left(c^{\prime}-c\right)\left(c^{\prime}-2 c\right)\left(c^{\prime}-3 c\right) \leqq 0,
\end{aligned}
$$

By a simple calculation Proposition 5.2 is concluded.

Remark. Example 2.5 gives an example of Proposition 5.2.

\section{Indefinite Einstein hypersurfaces.}

Indefinite Einstein hypersurfaces of $M^{\prime}=M_{s+t}^{n+1}\left(c^{\prime}\right)$ is said to be proper, if the shape operator is diagonalizable. By means of the rigidity theorem of complex submanifolds of an indefinite complex space form, Montiel and Romero [9] proved the following

THEOREM. (1) The proper complete simply connected indefinite complex Einstein hypersurfaces of $P_{s+t}^{n+1}(c)$ are only $P_{s}^{n}(c)$ with $t=0,1$ and $Q^{n}$ with $t=0$.

(2) In $H_{s+t}^{n+1}(c)$ they are only $H_{s}^{n}(c)$ with $t=0,1$ and $Q^{n}$ with $t=1$.

(3) In $C_{s+t}^{n+1}$ they are only $C_{s}^{n}$ with $t=0,1$.

This section is concerned with the local version of the above theorem. We prove here the following

THEOREM 6.1. Let $M$ be an indefinite complex Einstein hypersurface of $M_{s+t}^{n+1}\left(c^{\prime}\right)$. If $M$ is proper, then $M$ is totally geodesic or $S=n c^{\prime} g / 2$, the latter arising only when $c>0$ and $t=0$ or $c<0$ and $t=1$. 
Proof. Differentiating $\left(h_{\imath \bar{j}}\right)^{2}$ exteriorly twice, we get

$$
\sum \varepsilon \varepsilon_{r}\left(h_{\imath r k i} \bar{h}_{r j}+h_{\imath r k} \bar{h}_{r j l}\right)=0,
$$

where $\varepsilon=\varepsilon_{n+1}$, which implies $h_{2} h_{\imath j k i}=0$. Accordingly, it follows from this equation and (1.18) that

$$
h_{2}\left(n c^{\prime}-2 h_{2}\right)\left(\varepsilon_{k} h_{\imath j} \delta_{k l}+\varepsilon_{i} h_{j k} \delta_{i l}+\varepsilon_{j} h_{k i} \delta_{j l}\right)=0 .
$$

Since $M$ is proper, the second fundamental form $h_{\imath \jmath}$ is given by $\varepsilon_{i} \nu_{i} \delta_{i}$, and hence we have $h_{2}=\varepsilon \Sigma\left|\nu_{i}\right|^{2}$. It means that $h_{2}$ is non-positive or non-negative according as $\varepsilon=-1$ or $\varepsilon=1$ and $h_{2}=0$ holds true if and only if $M$ is totally geodesic.

Suppose that $h_{2}=n c^{\prime} / 2$. If $c^{\prime}>0$, then $h_{2}>0$ and hence we have $\varepsilon=1$ and $t=0$. On the other hand, if $c^{\prime}<0$, then $h_{2}<0$ and hence $\varepsilon=-1$ and $t=1$. Of course, the condition $h_{2}=n c^{\prime} / 2$ is equivalent to

$$
S_{\imath j}=n c^{\prime} \varepsilon_{i} \delta_{i j} / 2 \text {. }
$$

In the case where $h_{2} \neq 0$ or $n c^{\prime} / 2$, the equation (6.1) gives

$$
\varepsilon_{k} h_{\imath j} \delta_{k l}+\varepsilon_{i} h_{j k} \delta_{i l}+\varepsilon_{j} h_{k i} \delta_{j l}=0 \text {, }
$$

which yields that $h_{\imath \jmath}=0$. Thus $M$ is totally geodesic and one concludes the proof.

\section{Indefinite complex hypersurfaces with $R R=0$.}

This section is concerned with indefinite complex hypersurfaces with the Nomizu condition $R R=0$ of an indefinite complex Euclidean space $M^{\prime}=C_{s+t}^{n+1}$. Namely, it satisfies

$$
R(X, Y) R=0
$$

for any vector fields $X$ and $Y$ tangent to $M$. It turns out that the Nomizu condition implies $R S=0$ and moreover it is seen in [2] that in an indefinite hypersurface of an indefinite complex space form $M^{\prime}=M_{s+t}^{n+1}\left(c^{\prime}\right)$ if $c^{\prime} \neq 0$ and if $M$ satisfies $R S=0$, then $M$ is Einstein.

Now, suppose that $M$ is an indefinite complex submanifold satisfying the condition $R S=0$ of $M_{s+t}^{n+p}\left(c^{\prime}\right)$. It is seen that the condition $R S=0$ is equivalent to

$$
S_{i j k i}-S_{\imath j i k}=0 \text {, }
$$

and, combining (4.2) together with (1.16) and (1.17), we have

$$
c^{\prime}\left(\varepsilon_{i} \delta_{i l} S_{k j}-\varepsilon_{k} \delta_{k} S_{i i}\right) / 2-\sum \varepsilon_{r} \varepsilon_{x}\left(h_{i k}^{x} \bar{h}_{l r}^{x} S_{r j}-h_{k r}^{x} \bar{h}_{j l}^{x} S_{i \tilde{r}}\right)=0 .
$$

In particular, in an indefinite complex hypersurface of $C_{s+t}^{n+1}$, we have 


$$
h_{i k}\left(\bar{h}_{j l}\right)^{3}-\bar{h}_{j l}\left(h_{i k}\right)^{3}=0 \text {, }
$$

where we put $\left(h_{j k}\right)^{3}=\sum \varepsilon_{r} \varepsilon_{s} \varepsilon h_{j r} \bar{h}_{r s} h_{s k}$, which implies that

$$
\left(h_{j k}\right)^{3}=f h_{j k} \text { for a function } f \text { on } M \text {, }
$$

if the set consisting of points of $M$ at which the function $h_{2}$ is zero is measure zero. Under this hypothesis it follows that the equation (7.1) is equivalent to (7.2).

Remark. Taking account of (7.1), we remark here that if $c^{\prime}>0$ and if a space-like complex submanifold $M$ satisfies $R S=0$, then it is seen in [2] that $M$ is Einstein.

A complex hypersurface $M$ of index $2 s$ of $C_{s+t}^{n+1}$ is said to be cylindrical, if $M$ is a product manifold of $C_{a}^{n-1}$ and a complex curve in $C_{b}^{2}$ orthogonal to $C_{a}^{n-1}$ in $C_{s+t}^{n+1}(a+b=s+t)$. It is evident that a cylinder $M$ of index $2 s$ of $C_{s+t}^{n+1}$ satisfies (7.2), and hence it satisfies the condition $R S=0$, but it is not necessarily Einstein.

Remark. An example given in [1] is a complete indefinite complex hypersurface of $C_{s}^{2 n+1}$ which is Ricci-flat. Accordingly it satisfies $R S=0$ but it is not cylindrical. This is shown later. In a definite version, Takahashi [20] proved that the cylindrical hypersurface is the only complex hypersurface of $C^{n+1}$ satisfying the condition $R S=0$. However, as mentioned above, the property can not be extended in an indefinite Euclidean space.

In connection with cylindrical indefinite hypersurfaces, one poses the problem that "do there exist indefinite complex hypersurfaces satisfying $R S=0$ of $C_{s+t}^{n+1}$ which are not Einstein and not cylindrical ?"

In order to settle this problem affirmatively, an indefinite hypersurface satisfying the Nomizu condition of $M^{\prime}=C_{s+t}^{n+1}$ is considered. Let $M$ be an indefinite complex hypersurface of an indefinite complex Euclidean space $M^{\prime}=C_{s+t}^{n+1}$. By the twice exterior differentiation of the Riemannian curvature tensor $R$ the Ricci formula for $R$ is as follows:

$$
\begin{aligned}
& R_{\bar{i} j k \bar{l} m \bar{n}}-R_{\bar{\imath} j k \bar{i} \bar{n} m} \\
& =\sum \varepsilon_{r}\left(-R_{\bar{n} m r \bar{i}} R_{\bar{r} j_{k i}}+R_{\bar{n} m j \bar{r}} R_{\bar{i} r k \bar{l}}+R_{\bar{n} m k \bar{r}} R_{\bar{\imath} j r i}-R_{\bar{n} m r i} R_{\bar{i} j k \bar{r}}\right) .
\end{aligned}
$$

Taking account of the Gauss equation, we have

$$
\left\{\left(h_{m \bar{l}}\right)^{2} \bar{h}_{n i}+\left(h_{m \bar{\imath}}\right)^{2} \bar{h}_{n \ell}\right\} h_{j k}-\left\{\left(h_{k \bar{n}}\right)^{2} h_{m j}+\left(h_{j \bar{n}}\right)^{2} h_{m k}\right\} \bar{h}_{i l}=0,
$$

which implies following two equations:

$$
h_{i k}\left(\ddot{h}_{j l}\right)^{3}=\left(h_{i k}\right)^{3} \bar{h}_{j l},
$$




$$
h_{2}\left(h_{\imath j}\right)^{3}=h_{4} h_{\imath \jmath} \text {. }
$$

Making use of these results, one proves the following

Proposition 7.1. Let $M$ be an indefinite complex hypersurface of $M^{\prime}=C_{s+t}^{n+1}$. Then $M$ satisfies

$$
R(X, Y) R=0 \text { for any vector fields } X \text { and } Y
$$

if and only if

$$
h_{\imath j} h_{k l}=h_{i k} h_{j l} \text { or }\left(h_{\imath j}\right)^{2}=0 \text {. }
$$

Proof. Since the condition $R R=0$ is equivalent to (7.3), it is trivial that (7.6) is the sufficient condition for $R R=0$.

Conversely, combining the equation (7.3) together with (7.4) and (7.5), we have

$$
h_{2}\left\{\left(h_{m i}\right)^{2} \bar{h}_{n i}+\left(h_{m i}\right)^{2} \bar{h}_{n l}\right\}-2\left(h_{m \bar{n}}\right)^{4} \bar{h}_{i l}=0,
$$

and hence

$$
h_{4}\left\{h_{2}^{2}\left(h_{\imath j} h_{k l}+h_{i l} h_{j k}\right)-2 h_{4} h_{i k} h_{j l}\right\}=0 \text {. }
$$

As the above equation holds true for any indices $i, j, k$ and $l$, we have

$$
h_{4}\left(h_{2}{ }^{2}+2 h_{4}\right)\left(h_{\imath j} h_{k l}-h_{i k} h_{j l}\right)=0 \text {. }
$$

Suppose that there is a point $x$ at which $h_{4}=0$. When the point satisfies $h_{2} \neq 0,(7.5)$ implies $\left(h_{\imath \jmath}\right)^{3}=0$ and hence it follows from (7.3) that we have

$$
\left\{\left(h_{m i}\right)^{2}\left(h_{h i}\right)^{2}+\left(h_{m \bar{\imath}}\right)^{2}\left(h_{h i}\right)^{2}\right\}\left(h_{j \bar{p}}\right)^{2}=0,
$$

a contradiction. In this case, (7.7) implies $\left(h_{\imath j}\right)^{4}=0$ and when we put $\imath=l(7.3)$ is reduced to

$$
\left(h_{m \bar{l}}\right)^{2}\left(h_{i \bar{k}}\right)^{2}+\left(h_{m \bar{\imath}}\right)^{2}\left(h_{l \bar{k}}\right)^{2}-\left(h_{m l}\right)^{3} \bar{h}_{i l}=0 .
$$

Thus we have $\left(h_{j k}\right)^{3}=0$ and hence $\left(h_{j \bar{k}}\right)^{2}=0$. This completes the proof.

In a $(2 n+1)$-dimensional complex manifold $C^{2 n+1}$ with the standard basis, a Hermitian form $F$ is defined by

$$
F(z, w)=-\Sigma_{a} z_{a} \bar{w}_{a}+\Sigma_{x} z_{x} \bar{w}_{x}+\sum_{\jmath *} z_{j *} \bar{w}_{j *}+z_{2 n+1} \bar{w}_{2 n+1},
$$

where $a=1, \cdots, s ; x=s+1, \cdots, n$ and $j^{*}=n+j$ and $z=\left(z_{a}, z_{x}, z_{j *}, z_{2 n+1}\right)=$ $\left(z_{A}, z_{2 n+1}\right), w=\left(w_{A}, w_{2 n+1}\right)$ are in $C^{2 n+1}$. The scalar product defined by the real part $R e F$ is an indefinite Riemannian metric of index $2 s$ on $C^{2 n+1}$ and $\left(C^{2 n+1}, R e F\right)$ is a flat indefinite complex space form, which is denoted by $C_{s}^{2 n+1}$. Let $h$, be holomorphic functions of $C$ into $C$. For the complex coordinate 
system $\left(z_{A}, z_{2 n+1}\right)$ of $C_{s}^{2 n+1}$, let $M=M_{s}^{2 n}\left(h_{j} ; c_{j}\right)$ be the complex hypersurface of $C_{s}^{2 n+1}$ given by the equation

$$
z_{2 n+1}=\sum h_{j}\left(z_{j}+c_{j} z_{\jmath *}\right), \quad \jmath *=j+n
$$

for any complex number $c_{\jmath}$. Then it is seen in [1] that a vector $\xi$ at a point $z=\left(z_{A}, z_{2 n+1}\right)$ defined by

$$
\xi=\left(\bar{h}_{a}{ }^{\prime},-\bar{h}_{x}{ }^{\prime},-\bar{c}_{a} \bar{h}_{a *}{ }^{\prime},-\bar{c}_{x} \bar{h}_{x *^{\prime}}, 1\right)
$$

is normal to $M$ at $z$, and each $M_{s}^{2 n}\left(h_{\jmath} ; c_{\jmath}\right)$ is a complete complex hypersurface of index $2 n$ of $C_{s}^{2 n+1}$ if $\left|c_{a}\right| \geqq 1$ for any $a$. Furthermore, it is holomorphically diffeomorphic to $C^{2 n}$. The second fundamental form is also given by

$$
\begin{array}{ll}
h_{\imath \jmath}=\delta_{i j} h_{\imath}{ }^{\prime \prime} /|\xi|, & h_{\imath j *}=c_{i} \delta_{i j} h_{\imath}{ }^{\prime \prime} /|\xi|, \\
h_{i * \jmath}=c_{i} \delta_{i j} h_{\imath}{ }^{\prime \prime} /|\xi|, & h_{i * j *}=c_{\imath}{ }^{2} \delta_{i j} h_{\imath}{ }^{\prime \prime} /|\xi| .
\end{array}
$$

This means that it turns out that the first equation of (7.6) holds true, which implies that $M=M_{s}^{2 n}\left(h_{\jmath} ; c_{\jmath}\right)$ satisfies the Nomizu condition $R R=0$. Thus one finds

Proposition 7.2. There exist many indefinite complex hypersurfaces of $C_{s}^{2 n+1}$ for any $s$ with $R R=0$ which is not Einstein and not cylindrical.

\section{REFERENCES}

[1] R. Aiyama, T. IKawa, J.H. Kwon and H. Nakagawa, Complex hypersurfaces in an indefinite complex space form, Tokyo J. of Math., 10 (1987), 349-361.

[2] R. Aiyama, J.H. Kwon and H. Nakagawa, Complex submanifolds of an indefinite complex space form, J. Ramanujan Math. Soc., 2 (1987), 43-67.

[3] M. Barros And A. Romero, Indefinite Kähler manifolds, Math. Ann., 281 (1982), $55-62$.

[4] E. CALABI, Isometric imbedding of complex manifolds, Ann. of Math., 58 (1953), $1-23$.

[5] S. Y. Cheng AND S.T. YAU, Maximal space-like hypersurfaces in the LorentzMinkowski spaces, Ann. of Math., 104 (1976), 407-419.

[6] T. Ikawa, H. Nakagawa And A. Romero, Product complex submanifolds of indefinite complex space forms, to appear in Rocky Mountain J. Math.

[7] S. Kobayashi and K. Nomizu, Foundations of differential geometry, I, II, Interscience, 1963, 1969.

[8] M. Kon, Complex submanifolds with constant scalar curvature in a Kaehler manifold, J. Math. Soc. Japan, 27 (1975), 76-80.

[9] S. Montiel and A. Romero, Complex Einstein hypersurfaces of indefinite complex space form, Math. Proc. Camb. Phil. Soc., 94 (1983), 495-508.

[10] H. Nakagawa and K. OGiue, Complex space forms immersed in complex space forms, Trans. Amer. Math. Soc., 219 (1976), 289-297.

[11] H. NAKAgawa AND R. TAKAgI, On locally symmetric Kaehler submanifolds in a 
complex projective space, J. Math. Soc. Japan, 28 (1976), 638-667.

[12] S. Nishikawa, On maximal spacelike hypersurfaces in a Lorentzian manifold, Nagoya Math. J., 95 (1984), 117-124.

[13] H. OMORI, Isometric immersions of Riemannian manifolds, J. Math. Soc. Japan, 19 (1967), 205-211.

[14] A. Romero, Differential geometry of complex hypersurfaces in an indefinite complex space form, Univ. de Granada, 1986.

[15] A. Romero, On a certain class of complex Einstein hypersurfaces in indefinite complex space forms, Math. Z., 192 (1986), 627-635.

[16] A. Romero, Some examples of indefinite complex Einstein hypersurfaces not locally symmetric, Proc. Amer. Math. J. 98 (1986), 283-286.

[17] A. Romero, A class of complex hypersurfaces, Colloq. Math., 26 (1982), 175-182.

[18] A. Romero, An extension of Calabi's rigidity theorem to complex submanifolds of indefinite complex space form, Preprint.

[19] P. RyAN, Hypersurfaces with parallel Ricci tensor, Osaka J. Math., 8 (1971), 251259.

[20] T. TAkAhashi, Complex hypersurfaces with $R S=0$ in $C^{n+1}$, Tohoku Math. J., 25 (1973), 527-533.

[21] M. UMEHARA, Metric-preserving holomorphic mappings on indefinite Kaehler manifolds, Preprint.

[22] J. A. Wolf, Spaces of constant curvatures, McGrew-Hill, 1967.

[23] S. T. YAU, Harmonic functions on complete Riemannian manifolds, Comm. Pure and Appl. Math., 28 (1975), 201-228.

Institute of Mathematics UNIVERSITY OF TSUKUBA IBARAKI, 305

JAPAN
Department of Mathematics

Andong University

ANDONG, 760-380

KOREA 